\title{
Giant Dzyaloshinskii-Moriya interaction in Rashba superlattices
}

Woo Seung $\mathrm{Ham}^{1 \dagger}$, Abdul-Muizz Pradipto ${ }^{2,5 \dagger}$, Kay Yakushiji ${ }^{3}$, Kwangsu Kim ${ }^{4}$, Sonny S. Rhim $^{4}$, Kohji Nakamura ${ }^{2}$, Yoichi Shiota ${ }^{1}$, Sanghoon $\mathrm{Kim}^{4 *}$, and Teruo Ono ${ }^{{ }^{*}}$

1. Institute for Chemical Research, Kyoto University, Uji, Kyoto 611-0011, Japan

2. Department of Physics Engineering, Mie University, Tsu, Mie 514-8507, Japan

3. National Institute of Advanced Industrial Science and Technology (AIST), Tsukuba, Ibaraki 305-8568, Japan

4. Department of Physics, University of Ulsan, Ulsan 44610, South Korea

5. Faculty of Mathematics and Natural Sciences, Institut Teknologi Bandung, 40132 Bandung, Indonesia

${ }^{\dagger}$ These authors equally contributed to this work.

sanghoon.kim@ulsan.ac.kr, and ono@scl.kyoto-u.ac.jp

Dzyaloshinskii-Moriya interaction (DMI) is considered as one of the most important energy for specific chiral texture such as magnetic skyrmions. The key of generating DMI is absence of structural inversion symmetry and exchange energy with spin-orbit coupling. Therefore, a vast majority of researches about DMI is mainly limited to heavy metal/ferromagnet bilayer systems, only focusing on their interfaces. Here, we report that asymmetric band formation in an artificial superlattice arises from inversion symmetry breaking in stacking order of atomic layers, resulting in bulk DMI. Such bulk DMI is more than $\mathbf{3 0 0} \%$ larger than simple sum of interfacial contribution. Moreover, the asymmetric band is largely affected by strong spin-orbit coupling, showing crucial role of a heavy metal even in the non-interfacial origin of DMI. Such Rashba superlattices can be a new class of material design for spintronics applications. 


\section{Introduction}

The lack of inversion symmetry at the interface between a heavy metal (HM) and a ferromagnet (FM) induces the antisymmetric exchange interaction so-called Dzyaloshinskii-Moriya interaction (DMI) [1-4]. Recently, DMI has been intensively studied in the material combinations possessing perpendicular magnetic anisotropy (PMA) due to their necessities in creating magnetic chiral textures, such as magnetic skyrmions for the new type of racetrack memory device [5-8]. Generally, in order to stabilize skyrmions at room temperature, multilayer structures with repetitive stacking of FM/HM bilayer are utilized because multistacking of the bilayer unit easily provide the PMA and the sizable DMI at the same time, both of them arising from the same physical origin, i.e., interfacial SOC $[9,10]$. In this respect, $\mathrm{Co} / \mathrm{Pd}$ and $\mathrm{Co} / \mathrm{Pt}$ interfaces are one of the well-known material combinations providing both the PMA and the DMI originating from interfaces, resulting in stable magnetic skyrmions in $[\mathrm{Co} / \mathrm{Pd}]$ superlattices $[11,12]$. With the same manner of such a AB-type multi-stacking structure composed of several nanometer-thick layers as illustrated in Fig. 1(a, left), a superlattice with ABC-type repetitive stacking of a few atomic mono-layers [Fig. 1(b, right)] is interesting system. An epitome is the $[\mathrm{Co} / \mathrm{Pd} / \mathrm{Pt}]$ superlattice (SL) possessing PMA generated by the bulktype spin momentum locking due to absence of inversion symmetry in stacking order [13]. Note that not interfaces but asymmetry of bulk-type band formation in the $[\mathrm{Co} / \mathrm{Pd} / \mathrm{Pt}]-\mathrm{SL}$ as illustrated in Fig. 1(b) is essential to give rise to such a chiral phenomenon, resulting in strong PMA.

Such inversion symmetry breaking (ISB) in the SL with ABC-type stacking order would traditionally be accounted for by involving the Rashba model Hamiltonian, $\mathcal{H}_{R}=\alpha_{R}(\boldsymbol{k} \times \hat{z})$. $\boldsymbol{\sigma}$, which was initially proposed for a surface, where $\hat{z}$ is the direction of inversion-symmetrybreaking-induced potential gradient [14]. The oddness of the SOC in the $\boldsymbol{k}$ space due to the 
(a)

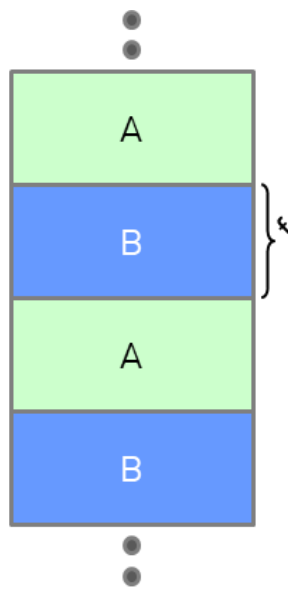

AB-type superlattice with $\mathrm{nm}$-thick layers

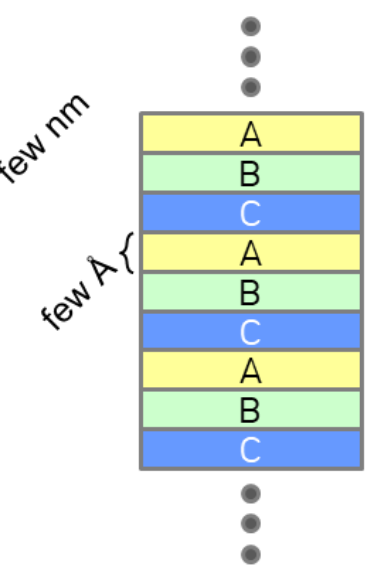

ABC-type superlattice with sub-nm-thick layers (b)

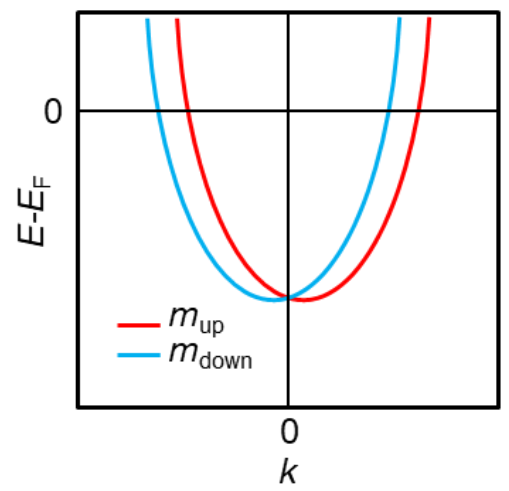

(c)

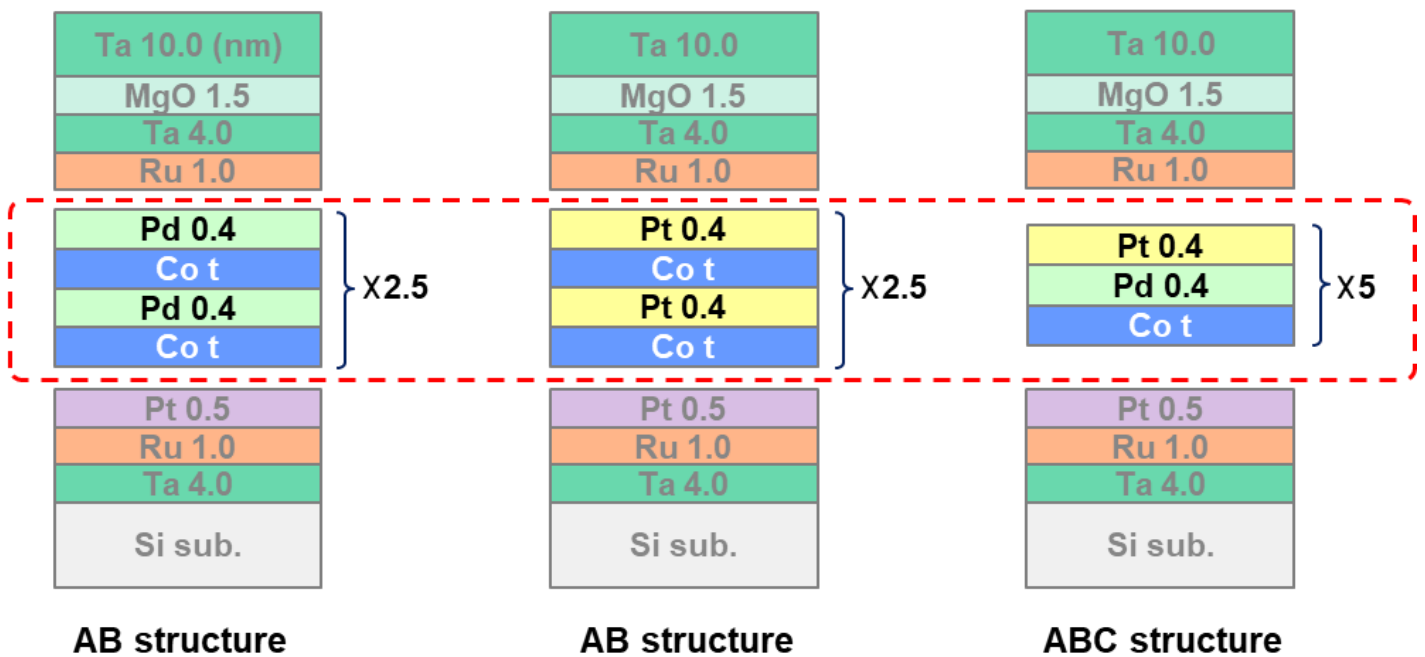

Figure 1. Superlattice structure with symmetry breaking in stacking order. Superlattices with (a, left) AB-type and (a, right) ABC-type stacking-order, which is composed of nm-thick and sub-nm-thick layers, respectively. (b) Asymmetry of band structure in terms of direction of magnetization in the superlattice with $\mathrm{ABC}$-stacking order. (c) Structures of the $[\mathrm{Co}(\mathrm{t}) / \mathrm{Pt}(0.4)]_{5},[\mathrm{Co}(\mathrm{t}) / \mathrm{Pd}(0.4)]_{5}$, and $[\mathrm{Co}(\mathrm{t}) / \mathrm{Pd}(0.4) / \mathrm{Pt}(0.4)]_{5}$-SL. For this study, the same buffer layer and capping layer structures (except the stacks in the red dotted box) were used for all samples.

ISB is shown by the dependence of the Hamiltonian on the linear terms in $\boldsymbol{k}$, although higher odd-order may in principle also appear. Rashba effect manifests most immediately into a spinsplitting within the $\boldsymbol{k}$ space, and it has been vastly utilized to interpret a number of magnetic phenomena [15-18], in particular those well understood to originate from the ISB, such as the Dzyaloshinskii-Moriya interaction responsible for exotic magnetic textures such as skyrmions and chiral domain walls, and spin-orbit torque. 
In this study, we investigate $\mathrm{DMI}$ of $[\mathrm{Co} / \mathrm{Pd} / \mathrm{Pt}]-\mathrm{SLs}$ arising from bulk spin-momentum locking. DMI of $[\mathrm{Co} / \mathrm{Pd} / \mathrm{Pt}]-\mathrm{SLs}$ with coherent lattice strain is about two-fold larger than those of other SL with inversion symmetry, $[\mathrm{Co} / \mathrm{Pd}]-$ and $[\mathrm{Co} / \mathrm{Pt}]-\mathrm{SLs}$. First-principles calculations reproduce such DMI enhancement in the ABC-type superlattice (hereafter Rashba superlattice), showing that the asymmetry of bands around Fermi energy level induced by ISB. Observed linear correlation of DMI with repetitions of the ABC-layer unit in the superlattice suggests that the enhancement of DMI is attributed to the bulk-type asymmetric band formation around the Fermi level.

\section{Results and Discussion}

\section{DMI of superlattices}

The magnetic superlattices of $[\mathrm{Co} / \mathrm{Pt}],[\mathrm{Co} / \mathrm{Pd}]$, and $[\mathrm{Co} / \mathrm{Pd} / \mathrm{Pt}]$ were prepared varying the $\mathrm{Co}$ thickness as shown in Fig. 1(c). Hereafter, [Co/Pd]-SL, [Co/Pd]-SL, and [Co/Pd/Pt]-SL. Firstly, the Co thickness dependence of DMI is investigated in $[\mathrm{Co}(\mathrm{t}) / \mathrm{Pt}(0.4)]_{5},[\mathrm{Co}(\mathrm{t}) / \mathrm{Pd}(0.4)]_{5}$, and $[\mathrm{Co}(\mathrm{t}) / \operatorname{Pd}(0.4) / \operatorname{Pt}(0.4)]_{5}-\mathrm{SL}$. In order to estimate the DMI energy of each superlattice, the measurement based on the extended droplet model was conducted [19,20]. Figure 2 (a) shows the schematic illustration of the droplet measurement. Since the nucleated magnetic droplet possesses domain wall magnetizations with two opposite radial direction. As illustrated in Fig. 2 (a), we can consider two domain wall magnetizations ( $\boldsymbol{M}_{\mathrm{DW} 1}$ and $\left.\boldsymbol{M}_{\mathrm{DW} 2}\right)$ with respect to $H_{\mathrm{x}}$. If we approximate that the two domain wall magnetizations dominate the total domain wall energy under $H_{\mathrm{x}}$, the total domain wall energy of nucleated magnetic droplet can be obtained from sum of the domain wall energies: $\sigma_{\text {total }}=\sigma_{\mathrm{DW} 1}\left(+\boldsymbol{H}_{\mathrm{x}}\right)+\sigma_{\mathrm{DW} 2}\left(-\boldsymbol{H}_{\mathrm{x}}\right)$, where $\sigma_{\mathrm{DW} 1}\left(+\boldsymbol{H}_{\mathrm{x}}\right)$ and $\sigma_{\mathrm{DW} 1}\left(-\boldsymbol{H}_{\mathrm{x}}\right)$ are the domain wall energies with respect to $H_{\mathrm{x}}$. The nucleation field through the relation as follows: $\mu_{0} \boldsymbol{H}_{\mathrm{n}}=\pi \sigma_{\mathrm{DW}, \text { total }}{ }^{2} t_{\mathrm{FM}} / 2 \mu_{0} M_{\mathrm{S}} p k_{\mathrm{B}} T$, where $\mathrm{t}_{\mathrm{FM}}$ is the ferromagnet thickness, $\mu_{0} \boldsymbol{M}_{S}$ is the saturation magnetization, $p$ represents the thermal stability 
(a)
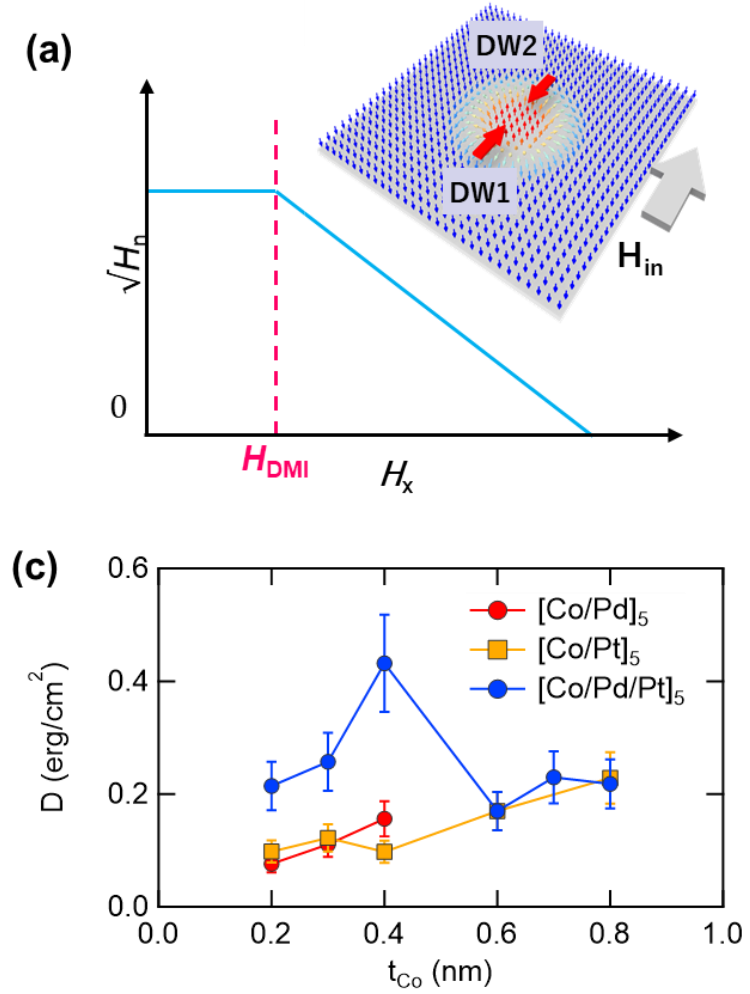

(d)

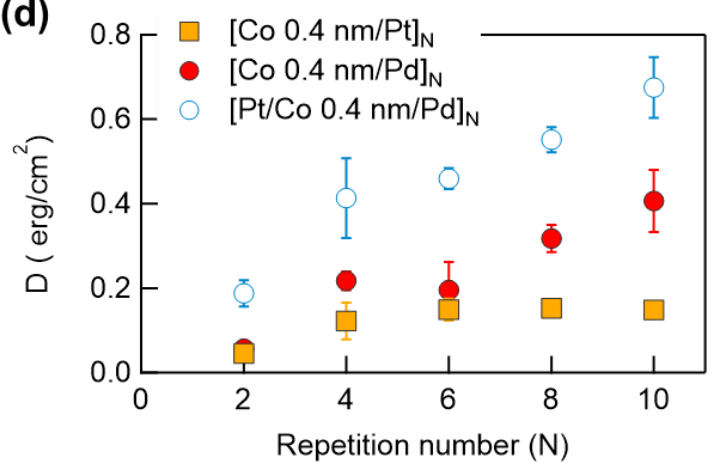

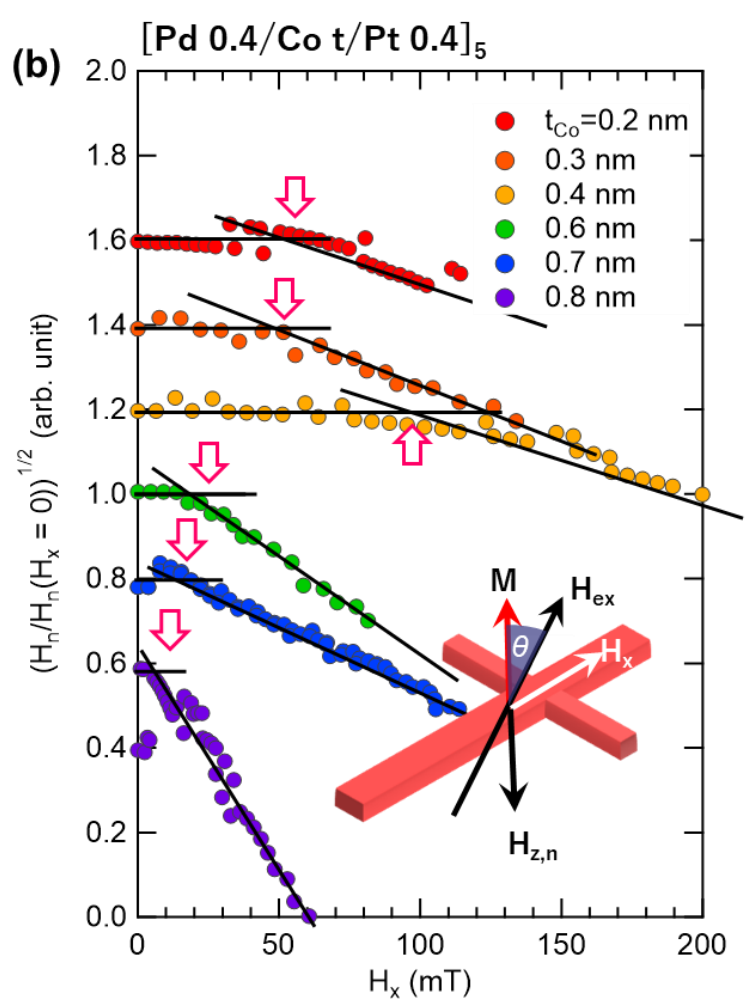

(e)

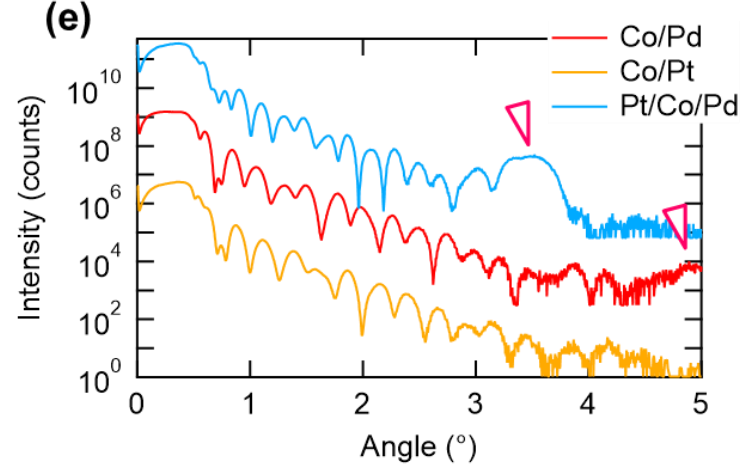

Figure 2. Large DMI of the Rashba SL. (a) Illustration of droplet nucleation field profile in terms of in-plane field. (b) Normalized $\sqrt{H_{n, z}}$ in terms of $H_{x}$. Inset shows the configuration of device with external field $\left(H_{e x}\right)$, components of the $H_{e x}\left(H_{x}\right.$ and $\left.H_{z}\right)$, and $M_{s}$. (c) $H_{\text {DMI }}$ and $D$ values of the SLs. (d) $D$ values of the SLs in terms of N. (e) XRR measurement results of SLs with $\mathrm{N}=10$.

factor, $k_{B}$ is the Boltzmann constant and $T$ denotes temperature [21]. Therefore, square root of nucleation fields in terms of $H_{\mathrm{x}}$ should follow the blue curve in Fig. 2 (a). Note that $\sigma_{\text {total }}$ is proportional to $\sqrt{\boldsymbol{H}_{\mathrm{n}, z}}$ when $\boldsymbol{H}_{\mathrm{x}}>\boldsymbol{H}_{\mathrm{DMI}}$, otherwise $\sigma_{\text {total }}$ becomes constant. The crossing point of the two linear curves can be defined as DMI-induced effective field $\left(\boldsymbol{H}_{\mathrm{DMI}}\right)$. Based on this model, the angular dependence of coercivity was measured to obtain $\sqrt{\boldsymbol{H}_{\mathrm{n}, z}}$ of the superlattices as illustrated in inset of Fig. 2 (b). In this measurement concept, applied magnetic 
field can be decomposed into $\boldsymbol{H}_{\mathrm{x}}$ and $\boldsymbol{H}_{\mathrm{n}, \mathrm{z}}$. The detailed measurement scheme is as follows; first of all, the sample is saturated to the $+\mathrm{z}$ direction. Then, the magnetic field is swept from the positive to negative field in terms of various polar angles $(\theta)$ in order to obtain the $\theta$ dependence of switching field $\left(\mu_{0} \boldsymbol{H}_{\mathrm{SW}}\right)$. Considering the measurement time scale (ramping rate $\sim 1 \mathrm{~T} / \mathrm{min}$.) is much slower than that of complete switching via domain wall propagation initiated from the nucleation, we can consider the relation of $\mu_{0} \boldsymbol{H}_{\mathrm{n}, \mathrm{z}}=\mu_{0} \boldsymbol{H}_{\mathrm{SW}} \cos \theta$. With this approach, we could obtain the quantitative information of DMI by plotting the $H_{x}$ dependence of $\sqrt{\boldsymbol{H}_{\mathrm{n}, \mathrm{Z}}}$.

In order to study the relation between DMI and the structural coherency, $\boldsymbol{H}_{\mathrm{DMI}}$ values of superlattices was obtained using above method in terms of $t_{\mathrm{Co}}$. The $\boldsymbol{H}_{\mathrm{x}}$ vs. $\sqrt{\boldsymbol{H}_{\mathrm{n}, z}}$ plots in Fig. 2 (b) displays that all $[\mathrm{Co} / \mathrm{Pd} / \mathrm{Pt}]_{5}$ films show the critical field which can be defined as $H_{\mathrm{DMI}}$ within the $t_{\text {Co }}$ range $0.2 \sim 0.8 \mathrm{~nm}$. This means Neel-type skyrmion-nucleation occurs in all films due to vertical ISB. Here, DMI energy density $(\boldsymbol{D})$ is discussed for excluding effects of $K_{\mathrm{eff}}$ and $M_{\mathrm{S}}$. $\boldsymbol{D}$ value can be estimated by using the equation of $\boldsymbol{D}=\mu_{0} \boldsymbol{M}_{\mathrm{S}} \Delta \boldsymbol{H}_{\mathrm{DMI}}$, where $\Delta$ denotes the domain wall width which is determined by the exchange stiffness constant $(A)$ and $K_{\text {eff }}$ by $\Delta=\sqrt{A / K_{\text {eff }}}$. In all samples, $A=10 p J / m$ is assumed. From D vs. $t_{\text {Co }}$ plots, we found that not only $[\mathrm{Co} / \mathrm{Pd} / \mathrm{Pt}]-\mathrm{SLs}$, but also other superlattices, $[\mathrm{Co} / \mathrm{Pt}]-\mathrm{SL}$ and $[\mathrm{Co} / \mathrm{Pd}]-\mathrm{SL}$, have sizable DMI although they are designed to have symmetric structure. It has been reported that a Co layer shares interfaces with top and bottom Pt or Pd layers, and sequential deposition process makes different interfacial qualities between $\mathrm{Co} / \mathrm{Pt}(\mathrm{Pd})$ and $\mathrm{Pt}(\mathrm{Pd}) / \mathrm{Co}$ interface $[22,23]$. That is to say, $[\mathrm{Co} / \mathrm{Pt}]-\mathrm{SL}$ and $[\mathrm{Co} / \mathrm{Pd}]-\mathrm{SL}$ also have structural asymmetry within out-of-plane direction. This will be discussed in detail at the end of this session.

Obtained $\boldsymbol{D}$ values of all superlattices are plotted in Fig. 2 (c). We can clearly see that $D$ of $[\mathrm{Co} / \mathrm{Pd} / \mathrm{Pt}]-\mathrm{SLs}$ is $3 \sim 4$ times larger than those of other superlattices when $t_{\mathrm{Co}}<0.6 \mathrm{~nm}$, while 
$H_{\mathrm{DMI}}$ values become similar to each other when $t_{\mathrm{Co}}>0.4 \mathrm{~nm}$. We found that range of the $t_{\mathrm{Co}}$ under $0.6 \mathrm{~nm}$ is coherent strain regime, and the ABC-type structure shows exceptionally larger $K_{\text {eff }}$ only in the thickness range [Supplementary Information]. A possible origin of such large anisotropy in the $[\mathrm{Co} / \mathrm{Pt} / \mathrm{Pd}]-\mathrm{SL}$ is bulk spin momentum locking arising from the symmetry breaking due to odd contribution of magnetic anisotropy energy [18]. In this point of view, large $\mathrm{DMI}$ in the $[\mathrm{Co} / \mathrm{Pd} / \mathrm{Pt}]-\mathrm{SL}$ within the coherent strain regime $\left(t_{\mathrm{Co}} \leq 0.4 \mathrm{~nm}\right)$ is also due to the bulk-spin momentum locking arising from symmetry breaking with stacking order in the superlattice.

Note that difference of DMI between $\mathrm{Pt} / \mathrm{Co}$ and $\mathrm{Co} / \mathrm{Pd}$ interfaces in the $[\mathrm{Co} / \mathrm{Pd} / \mathrm{Pt}]-\mathrm{SL}$ can also enhance total DMI energy and the value should be larger than what other symmetric structures have. In other words, the interfacial origin should be also considered to explain our observation. In order to confirm the characteristics of DMI originating from the interface, the dependence of repetition number $(\mathrm{N})$ was studied in all series of the SLs. Figure 2 (d) shows that both the $[\mathrm{Co} / \mathrm{Pd} / \mathrm{Pt}]-\mathrm{SL}$ and the $[\mathrm{Co} / \mathrm{Pd}]-\mathrm{SL}$ show repetition number $(\mathrm{N})$ dependence of DMI, while the $[\mathrm{Co} / \mathrm{Pt}]-\mathrm{SL}$ does not. Especially, DMI of the $[\mathrm{Co} / \mathrm{Pd} / \mathrm{Pt}]-\mathrm{SL}$ linearly increases by more than $300 \%$ when $\mathrm{N}$ increases from 2 to 10 . We note that such linear dependence of DMI cannot be simply explained by the interfacial origin. If the increase in DMI with repetition numbers is fully originated from the interfacial spin-orbit coupling, the DMI should be independent with the repetition numbers because the gains in the DMI energy by increasing the number of interfaces are compensated by the volume of Co layers. We note that $[\mathrm{Pd} / \mathrm{Co} / \mathrm{Pd}]-\mathrm{SL}$ also shows $\mathrm{N}-$ dependence of DMI with experiment in spites of the inversion symmetry. Since the $\mathrm{Co} / \mathrm{Pd}$ interface can be easily intermixed more than a $\mathrm{Pd} / \mathrm{Co}$ because $\mathrm{Pd}$ is heavier than Co [22, 23], different level of intermixing at $\mathrm{Pd} / \mathrm{Co}$ and $\mathrm{Co} / \mathrm{Pd}$ interfaces induces ISB, thereby sizable DMI can be generated. Therefore, $[\mathrm{Co} / \mathrm{Pd} / \mathrm{Pt}]-\mathrm{SL}$ and $[\mathrm{Pd} / \mathrm{Co}]-\mathrm{SL}$ may share the same origin to have $\mathrm{N}$ - 
dependence of DMI. Observation of skyrmion phase in the $\mathrm{Co} / \mathrm{Pd}$ multilayers has been reported based on this scenario [11]. Though the same thing can occur in the $[\mathrm{Pt} / \mathrm{Co} / \mathrm{Pt}]-\mathrm{SL}$, there is no Ndependence of DMI possibly due to that Pt is heavier than Pd. In other words, severe intermixing between Co and Pt may induce random arrangement of atoms during the deposition process, resulting in decrease of DMI. The x-ray reflectivity (XRR) study shown in Fig. 2 (e) clarifies this point. The $1^{\text {st }}$-Bragg-like peak in XRR is known to be observed in the superlattice possessing periodicity [24]. In case of $[\mathrm{Co} / \mathrm{Pd} / \mathrm{Pt}]-\mathrm{SL}$ and $[\mathrm{Co} / \mathrm{Pd}]-\mathrm{SL}$, the $1^{\text {st }}$-Bragg-like peaks are shown in the XRR spectra, while there is no indication of the Bragg-like peak for [Co/Pt]-SL, implying that there is an intermixing at the interface $[24,25]$. Therefore, the observed DMI of [Co/Pt]-SL should arise from the interface between randomly intermixed SL and bottom Pt buffer layer. On the other hands, not only $[\mathrm{Co} / \mathrm{Pd} / \mathrm{Pt}]-\mathrm{SL}$, but also $[\mathrm{Co} / \mathrm{Pd}]-\mathrm{SL}$ should have structural asymmetry, as confirmed by the periodicity in XRR results. Our results imply that such a structural asymmetry is essential for the $\mathrm{N}$ dependence of DMI. In order to understand the correlation between structural asymmetry and N-dependence of DMI under microscopic point of view, we conducted the $1^{\text {st }}$ principle calculation as explained in following section.

\section{Theoretical Consideration about DMI of the Superlattices}

Estimation of the magnetocrystalline energy (MCA) and DMI is done by following the steps outlined previously $[13,26]$ Here, we consider $1 \sim 4[\mathrm{Co} / \mathrm{Pt} / \mathrm{Pd}]$ units, and anticlockwise rotation of the spin spiral structures as shown in Fig. 3(a)-(c). Detail process is explained in the Method Section. The calculation results are summarized in Fig. 3 (d) and (e). The odd terms of the MCA energy $\left(E_{M C A}^{\text {odd }}\right)$, which quantifies ISB, are summarized in Fig. 3(d). This quantity is related to the ISB-induced shift of the band structure along the $k_{y}$ direction due to the magnetization along $x$ direction. We note that $E_{M C A}^{\text {odd }}$ increases with the repetition number $\mathrm{N}$ of $[\mathrm{Co} / \mathrm{Pt} / \mathrm{Pd}]$ unit layers.

The total MCA and $E_{M C A}^{o d d}$ in the $[\mathrm{Pt} / \mathrm{Co} / \mathrm{Pd}]_{n}$ increases shown in Fig. 3(d), and the total MCA 


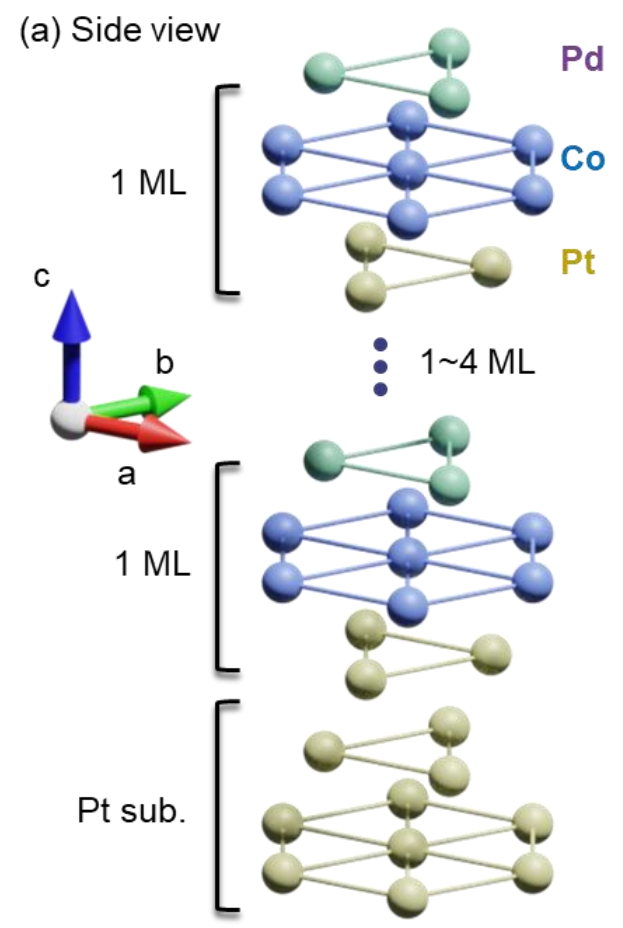

(b) Top view

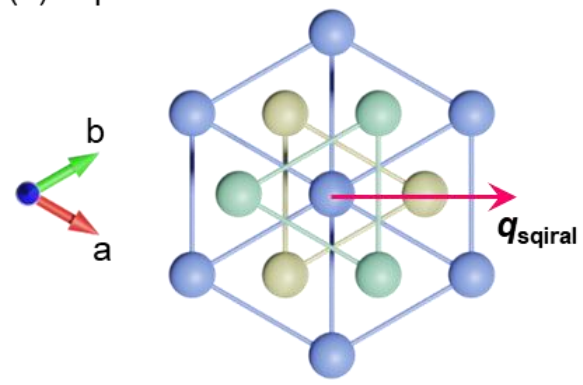

(c)

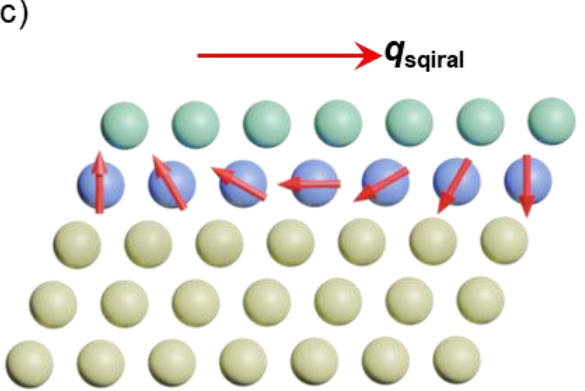

(d)

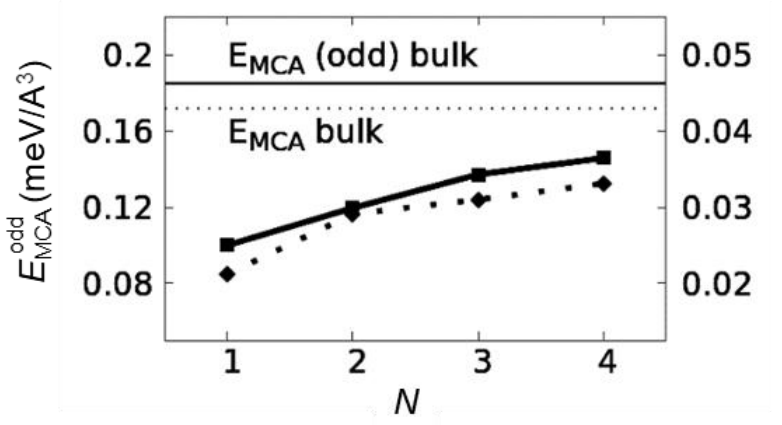

(e)

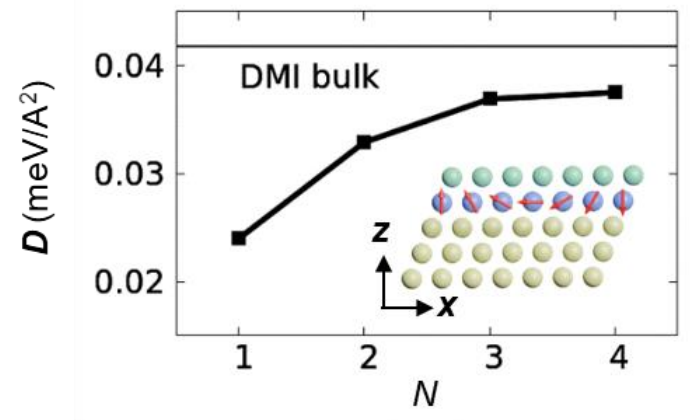

Figure 3. ISB-related MCA energy and DMI of the [Co/Pt/Pd]-SL. (a) The side view and (b) the top view of the model crystal structure of $\mathrm{Co} / \mathrm{Pt} / \mathrm{Pd}$-based multilayer system. The $\boldsymbol{M}$ direction for the propagation of the spin-spiral wave is indicated by the red arrow in (b). (c) Anticlockwise rotation of the spin spiral structures. The calculated N dependence of (d) the odd term of MCA energies $E_{M C A}^{\text {odd }}$ and (e) the DMI for the $[\mathrm{Pt} / \mathrm{Co} / \mathrm{Pd}]_{\mathrm{n}}$ model systems, while those obtained for the bulk system are shown using straight lines. The $\mathrm{N}$ dependence of the total MCA energies $E_{M C A}$ of the $[\mathrm{Pt} / \mathrm{Co} / \mathrm{Pd}]_{\mathrm{n}}$ model systems and the corresponding bulk value are shown in (a) using dashed lines.

value can be expected to reach $0.043 \mathrm{meV} / \AA^{2}$ obtained for the bulk $[\mathrm{Co} / \mathrm{Pt} / \mathrm{Pd}]-\mathrm{SL}$, i.e. the infinitely periodic system along $c$ direction $[\mathrm{Pt} / \mathrm{Co} / \mathrm{Pt}]_{\infty}[27]$. When the DMI constants are further extracted by utilizing the polynomial expression of the frozen magnon energy (Eq. S1 in Supplementary Information), we obtain $\boldsymbol{D}$ value of the asymmetric $[\mathrm{Pt} / \mathrm{Co} / \mathrm{Pd}]_{\mathrm{N}}$ structure which increases with $\mathrm{N}$ [see Fig. 3(e)]. At this stage, a quantitative agreement with the measured DMI 
for these systems is difficult due in parts to the idealized structures, i.e. without in-plane structural asymmetry, used in calculations. However, the increase of DMI in the $[\mathrm{Pt} / \mathrm{Co} / \mathrm{Pd}]_{\mathrm{N}}$ is fully consistent with our experimental observations. Atomic intermixing should be also considered in calculations as observed in our experiments. However, both studies manifest that DMI in such an ABC-type structure with coherent strain cannot be explained with conventional approach with interfacial origin.

The two-dimensional MCA contour map of $[\mathrm{Pt} / \mathrm{Co} / \mathrm{Pd}]_{1}$ is shown in Fig. 4(a). This map shows the typical characteristic of the MCA in the systems with ISB. Since the in-plane magnetization is oriented along the $+\mathrm{x}$ direction, i.e. $\boldsymbol{m}=m_{+x} \hat{x}$, the MCA is asymmetric along the $k_{y}$ axis, which is along the $\hat{z} \times \boldsymbol{m}$ direction, and shows mirror symmetry along $k_{x}$ direction. Similar behavior is obtained for other N. The Fermi surface of $[\mathrm{Pt} / \mathrm{Co} / \mathrm{Pd}]_{1}$ is visibly shifted towards negative $k_{y}$ as shown by using the red color in Fig. 4(b) for $m_{\mathrm{+}_{\mathrm{x}}}$ magnetization, and is shifted symmetrically towards negative $k_{y}$ when the magnetization is along the $m_{-x} \hat{x}$ direction, as plotted by using the blue color in Fig. 4(b). In order to check the band contribution to the Rashba splitting, we plot Fermi surface by switching off the spin-orbit coupling in each atomic layer. Comparing the results shown in Figs. 4(d)-(f), it is visible that switching off SOC in Pt layer alters the shape of the Fermi contour most considerably compared to Co and Pd, implying crucial role of Pt bands to the band splitting, which should be attributed to the strong SOC constant of the Pt atom.

The anatomy of the DMI can be decomposed in a similar fashion. For this purpose, one can extract the DMI using the polynomial expression (Eq. 1 in Method Section) from the spiral structures built for several $q$ vectors by switching on/off the SOC of a particular layer. In this work, however, we choose a simpler approach, i.e. by calculating the asymmetry between the energies of spiral structures with $q= \pm 0.25$, of which the degeneracy is lifted due to the ISB. When the asymmetric energy in a total structure, which is correlated with DMI and defined here as the energy difference between 
$q= \pm 0.25$ states, are denoted as $E_{q= \pm 0.25}^{a s y m}$, th e contribution of a particular layer $L$ to the asymmetric energy can be given as

$$
E_{q= \pm 0.25}^{a s y m}(L)=E_{q= \pm 0.25}^{a s y m}(\text { tot })-E_{q= \pm 0.25}^{a s y m}\left(L_{\mathrm{off}}\right)
$$

where $E_{q= \pm 0.25}^{\text {asym }}($ tot $)$ is the asymmetric energy which includes the contribution of all layers, and $E_{q= \pm 0.25}^{\text {asym }}\left(L_{\text {off }}\right)$ is the asymmetric energy when the SOC contribution of layer $L$ is switched off. The results are summarized in Fig. 5, clearly showing that, first of all, despite being the carrier of the magnetic moments, Co gives very small contribution to the DMI. In fact, the largest contribution to the asymmetric energy, hence to the DMI, is coming from the neighboring Pt layers,
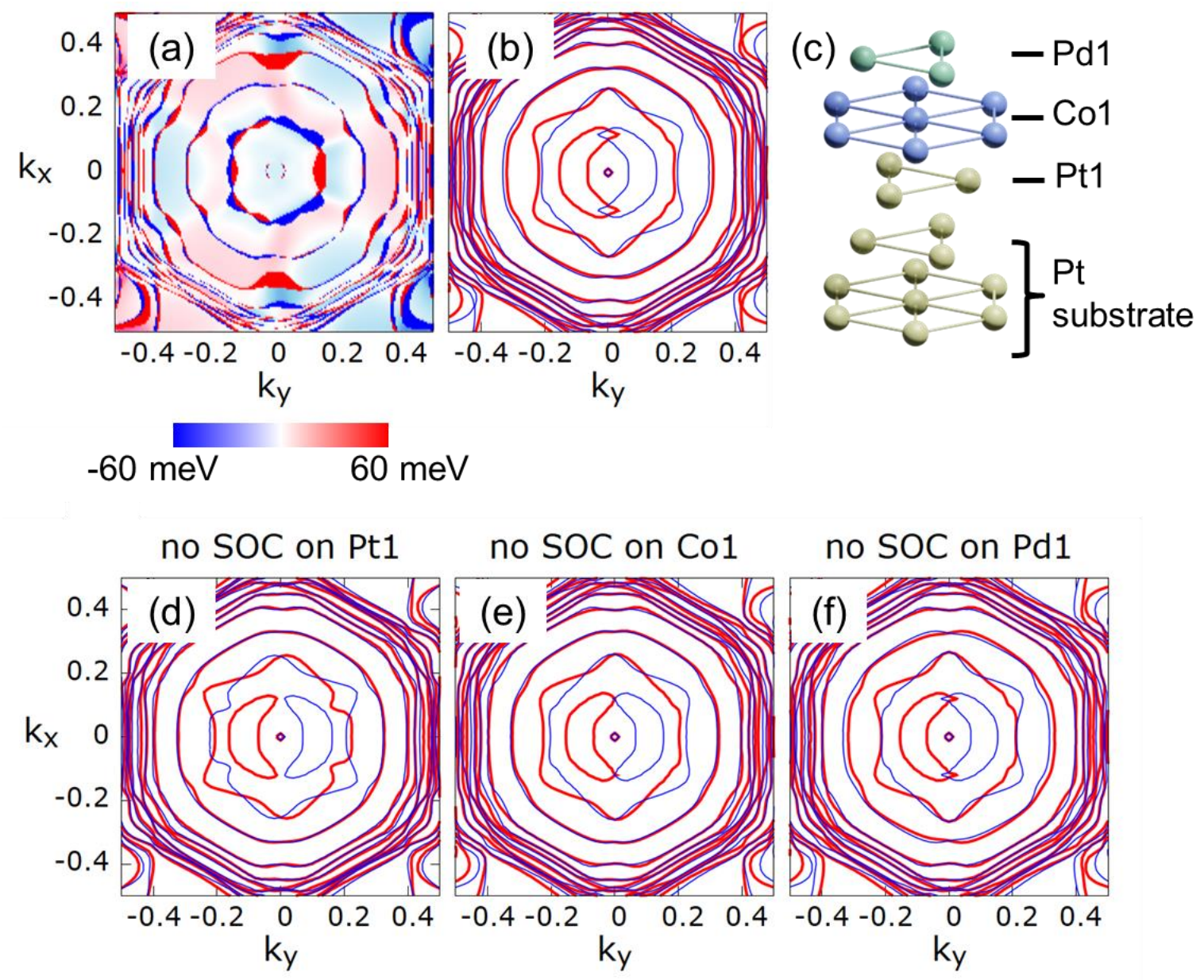

Figure 4. $k$-dependent MCA energy and Fermi surface in the $[\mathrm{Pt} / \mathrm{Co} / \mathrm{Pd}]$ unit structure. (a) Two dimensional $\left(k_{\mathrm{x}}, k_{\mathrm{y}}\right)$ contour map of $\boldsymbol{k}$-dependent MCA energy and (b) Fermi surface for different inplane magnetization directions along $+\mathrm{x}$ and $-\mathrm{x}$ axes, plotted using respectively red and blue lines, of the $[\mathrm{Pt} / \mathrm{Co} / \mathrm{Pd}]_{1}$ model system depicted in (c). (d)-(f) Fermi surface of the $[\mathrm{Pt} / \mathrm{Co} / \mathrm{Pd}]_{1}$ model system in the absence of SOC on the Pt1, Co1, and Pd1 layer, respectively. 


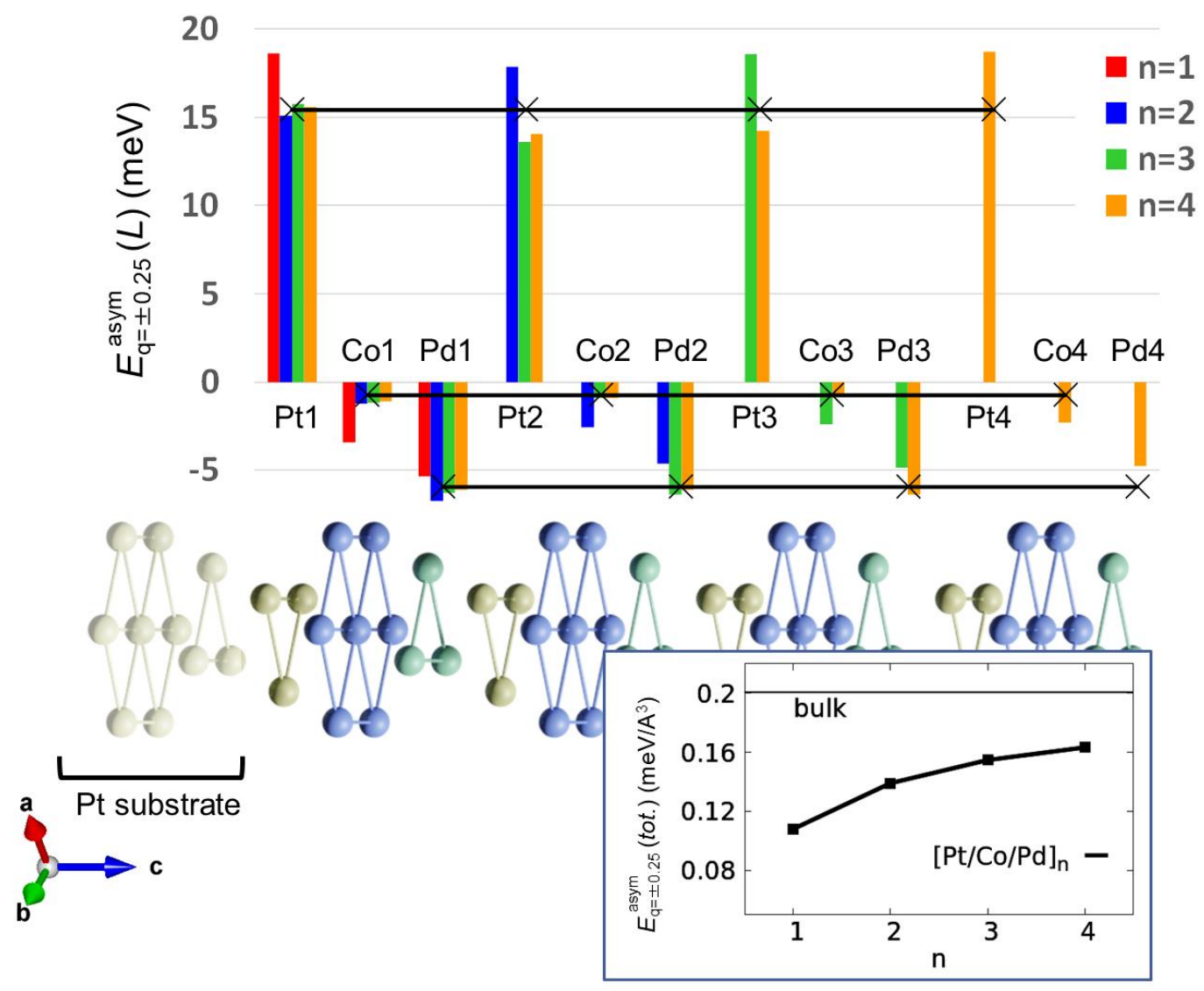

Figure 5. Origin of the $\mathbf{N}$-dependence of $\mathrm{DMI}$ in the $[\mathrm{Pt} / \mathrm{Co} / \mathrm{Pd}]-\mathrm{SL}$. The contribution of different atomic layer $L$ to the asymmetric energy between the spiral structures with wave vectors $q= \pm 0.25$, $E_{q= \pm 0.25}^{\text {asym }}(L)$. The corresponding atomic layer contributions for bulk system, i.e. with $\mathrm{n}=\infty$, are shown with black lines. The sequence of the layers is illustrated. The total asymmetric energy $E_{q= \pm 0.25}^{a s y m}$ (tot) is also shown.

which can be understood to dominate the band around Fermi level, as shown in Figs. 4(d)-(f). It should be noted that for all N, Pd gives smaller and opposite contribution to the DMI, in comparison to Pt. Additionally, the evaluated total $E_{q \pm 0.25}^{a s y m}$ for all N, as plotted in the inset of Fig. 5, shows considerable deviations for all contributions, then, converges to that of the infinitely periodic bulk. This implies a non-interfacial origin, but bulk spin-momentum locking for the DMI in the considered $[\mathrm{Pt} / \mathrm{Co} / \mathrm{Pd}]-\mathrm{SL}$ systems ${ }^{30}$.

\section{Conclusion}

Our experimental and theoretical studies demonstrate that the bulk spin-momentum locking in 
a superlattice can be made with asymmetric atomic stacking and structural coherency. Especially, the N-dependence of DMI of the $[\mathrm{Co} / \mathrm{Pd} / \mathrm{Pt}]-\mathrm{SL}$ is important phenomenon arising from the band asymmetry. Because interfacial DMI in a ferromagnet/heavy metal bilayer has been only considered so far, material selection has been limited to several cases for development of skyrmion-based devices. On the other hand, bulk DMI can be made with such ABC-type material combination within atomic scale, which is larger than interfacial contribution. Our experimental and theoretical findings can provide a new class of material design for spintronic devices with chiral magnets.

\section{References}

[1] Dzyaloshinskii, I. E. Thermodynamic theory of "Weak" ferromagnetism in antiferromagnetic substances. Sov. Phys. JETP. 5, 1259 (1957).

[2] Moriya, T. New Mechanism of Anisotropic Superexchange Interaction. Phys. Rev. Lett. 4, $228-230(1960)$.

[3] Moriya, T. Anisotropic Superexchange Interaction and Weak Ferromagnetism. Phys. Rev. 120, $91(1960)$.

[4] Heide, M., Bihlmayer, G. \& Blügel, S. Dzyaloshinskii-Moriya interaction accounting for the orientation of magnetic domains in ultrathin films: Fe/W(110). Phys. Rev. B 78, 140403 (2008).

[5] Fert, A., Cros, V.\& Sampaio, J. Nat. Nanotechnol. 8, 152-156(2013).

[6] Emori, S., Bauer, U., Ahn, S.-M., Martinez, E. \& Beach, G. S. D. Current-driven dynamics of chiral ferromagnetic domain walls. Nat. Mater. 12, 611-616 (2013).

[7] Boulle, O. et al. Room temperature chiral magnetic skyrmions in ultrathin magnetic nanostructures. Nat. Nanotechnol. 11, 449-454 (2016). 
[8] Song, K. M. et al. Skyrmion-based artificial synapses for neuromorphic computing. Nat. Elec. 3, 148-155 (2020).

[9] Woo, S. et al. Observation of room-temperature magnetic skyrmions and their currentdriven dynamics in ultrathin metallic ferromagnets. Nat. Mater. 15, 501-506 (2016).

[10] Jiang, W. et al. Skyrmions in magnetic multilayers. Phys. Rep. 704, 1-49 (2017).

[11] Pollard, S. D. et al. Observation of stable Neel skyrmions in cobalt/palladium multilayers with Lorentz transmission electron microscopy. Nat. Comm. 8, 14761 (2017)

[12] Gilbert, D. A. et al. Realization of ground-state artificial skyrmion lattices at room temperature. Nat. Comm. 6, $8462(2015)$.

[13] Pradipto, A.-M. et al. Enhanced perpendicular magnetocrystalline anisotropy energy in an artificial magnetic material with bulk spin-momentum coupling. Phys. Rev. B 99, 180410 (2019).

[14] Bychkov, Y. A. \& Rashba, E. I., Properties of a 2D electron gas with lifted spectral degeneracy. JETP Lett. 39, 78 (1984).

[15] Kim, K.-W., Lee, H.-W., Lee, K.-J. \& Stiles, M. D., Chirality from Interfacial Spin-Orbit Coupling Effects in Magnetic Bilayers. Phys. Rev. Lett. 111, 216601 (2013).

[16] Barnes, S., Ieda, J. \& Maekawa, S. Rashba Spin-Orbit Anisotropy and the Electric Field Control of Magnetism. Sci. Rep. 4, 4105 (2015).

[17] Yang, H., Vu, A. D., Hallal, A, Rougemaille, N., Coraux, J., Chen, G., Schmid, A. K., \& Chshiev, M., Anatomy and giant enhancement of the perpendicular magnetic anisotropy of cobalt-graphene Heterostructures. Nano Lett. 16, 145 (2016).

[18] Yang, H., et al. Significant Dzyaloshinskii-Moriya interaction at graphene-ferromagnet interfaces due to the Rashba effect. Nature Mater 17, 605-609 (2018).

[19] Kim, S. et al. Magnetic droplet nucleation with a homochiral Neel domain wall. Phys. Rev. B., 95, 220402 (2017) 
[20] Kim, S. et al. Correlation of the Dzyaloshinskii-Moriya interaction with Heisenberg exchange and orbital asphericity, Nat. Commun. 9, 1648 (2018).

[21] Pizzini, S. et al. Chirality-Induced asymmetric magnetic nucleation in $\mathrm{Pt} / \mathrm{Co} / \mathrm{AlOx}$ ultrathin microstructures. Phys. Rev. Lett. 113, 047203 (2014).

[22] Maesaka, A. and Ohmori, H. Transmission electron microscopy analysis of lattice strain in epitaxial Co-Pd multilayers. IEEE Trans. Magn. 38, 2676-2678 (2002).

[23] Jamali, M. et al. Spin-orbit torques in Co/Pd multilayer nanowires. Phys. Rev. Lett. 111, 246602 (2013).

[24] Yao, B, Coiffey, Kevin. R., The influence of periodicity on the structures and properties of annealed $[\mathrm{Fe} / \mathrm{Pt}]_{\mathrm{n}}$ multilayer films. J. Mag. Mag. Mater. 320, 559 (2008).

[25] Yu, Y. S. et al. Structure and magnetic properties of magnetron-sputtered $\mathrm{FePt} / \mathrm{Au}$ superlattice films. J. Phys. D; Appl. Phys. 41, 245003 (2008).

[26] Nakamura, K., Ito, T., Freeman, A. J., Zhong, L. \& Fernandez-de-Castro, Enhancement of magnetocrystalline anisotropy in ferromagnetic Fe films by intra-atomic noncollinear magnetism. Phys. Rev. B 67, 014420 (2003).

[27] Rado, G. T. and Suhl, H. Herring, C. Exchange Interactions Among Itinerant Electrons (Academic Press, 1966).

[28] Yamamoto, K., et al. Interfacial Dzyaloshinskii-Moriya interaction and orbital magnetic moments of metallic multilayer films. AIP Adv. 7, 056302 (2017).

[29] Nakamura, K., et al. Symmetric and asymmetric exchange stiffnesses of transition-metal thin film interfaces in external electric field. J. Magn. Magn. Mater. 457, 97 (2018).

[30] Mohammad Saeed Bahramy and Naoki Ogawa, Bulk Rashba Semiconductors and Related Quantum Phenomena, Adv. Mater. 29, 1605911 (2017). 


\section{Methods}

\section{Experiment}

The magnetic superlattice structures of $[\mathrm{Co} / \mathrm{Pt}],[\mathrm{Co} / \mathrm{Pd}]$, and $[\mathrm{Co} / \mathrm{Pd} / \mathrm{Pt}]$ were deposited by the UHV magnetron sputtering system. Hereafter, $[\mathrm{Co} / \mathrm{Pd}]-\mathrm{SL},[\mathrm{Co} / \mathrm{Pd}]-\mathrm{SL}$, and $[\mathrm{Co} / \mathrm{Pd} / \mathrm{Pt}]-\mathrm{SL}$. The thickness of magnetic layer (Co) is varied in each superlattice. The static magnetic properties such as saturation magnetization $\left(\boldsymbol{M}_{\mathrm{S}}\right)$ and magnetic anisotropy energy are investigated by the vibrating sample magnetometer (VSM). In order to quantify DMI energy, we used the extended droplet method [19-21]. All the films were patterned into a microstrip with a Hall bar structure by E-beam lithography to prevent the nucleation of domain at the rough microstrip edge. $\mathrm{Ti}(5 \mathrm{~nm}) / \mathrm{Au}(100 \mathrm{~nm})$ electrodes are defined to make electrical contacts with $\mu \mathrm{m}$-scale Hall bars by photolithography and lift-off process.

\section{The $1^{\text {st }}$ principle calculation for MCA and DMI of SLs}

The MCA energy $E_{\mathrm{MCA}}$ has been defined in our calculation as the energy difference between the in- and out-of-plane magnetization direction, i.e. $E_{\mathrm{MCA}}=E_{\mathrm{ip}}-E_{\mathrm{op}}$, where $E_{\mathrm{ip}}$ and $E_{\mathrm{op}}$ refer respectively to the total energy of the in- and out-of-plane magnetization. The inclusion of SOC is done via the second variational method [5]. The in-plane magnetization direction has been chosen to be along the $\mathrm{x}$ direction. We found that our calculated MCA energy has converged at a relatively large two-dimensional $\mathrm{k}$-point mesh of $100 \times 100$. In addition to the total MCA energy, we also virtually decomposed the k-dependent MCA energy into $E_{\mathrm{MCA}}^{+k_{y}}$ and $E_{\mathrm{MCA}}^{-k_{y}}$ in which $E_{M C A}^{ \pm k_{y}}=E\left(m_{\mathrm{x}}, \pm k_{\mathrm{y}}\right)-E\left(m_{\mathrm{z}}, \pm k_{\mathrm{y}}\right)$ and calculated the odd term of the MCA energy $E_{\mathrm{MCA}}^{\text {odd }}$ by following the recipe in our previous work [13], as $E_{M C A}^{\text {odd }}=E_{M C A}^{+k_{y}}-E_{M C A}^{-k_{y}}$. This quantity, despite the fact that it contains no physical meaning, illustrates the physics of Rashba spin-orbit coupling and provides an estimation of the degree of ISB by the shift of the Fermi surface. Comparison with bulk values has been done with a three-dimensional k-point mesh of 75 
$\times 75 \times 45$ for the bulk [Co/Pt/Pd]-SL [13]

The estimation of $\boldsymbol{D}$ values was done by following the steps outlined previously [13, 26]. We start from the ferromagnetic configuration which is found to be the ground state of all model systems at the scalar relativistic approximation, i.e. without the SOC. Next a set of spiral spin structures with wave vectors $\boldsymbol{q}=a / \lambda$ along the $M=(1,1,0)$ direction (see Fig. $3 \mathbf{b}$ ), where $\lambda$ is the wavelengths of the spin spiral structures, are generated by utilizing the generalized Bloch theorem $[28,29]$. When the SOC is then included, the spin-spiral structures have been assumed to be the Néel $x z$ out-of-plane rotation type. The frozen magnon energy, $E(q)$, for $q=0, \pm 0.1$, $\pm 0.2, \pm 0.25$ has been fitted with a polynomial expansion;

$$
E(q)=C_{0}+C_{1} q+C_{2} q^{2}+C_{3} q^{3}+C_{4} q^{4}
$$

where the odd terms $C_{1}$ and $C_{3}$ occur due to the presence of ISB. The DMI discussed in this work is extracted as the antisymmetric exchange stiffness constant $C_{1}$, i.e. first order in $q$, or $\boldsymbol{D} \equiv C_{1}$. Convergence of the calculated DMI has been obtained with a $40 \times 40 \mathrm{k}$-point mesh within the two-dimensional Brillouin zone. As in the case of MCA energy, we have also computed the DMI for the bulk CoPtPd system with the Néel $x z$ out-of-plane rotation type magnetic structures along the $\boldsymbol{M}$ direction, and a three-dimensional $40 \times 40 \times 20 k$-point mesh has been used.

\section{Acknowledgement}

Financial support from the Japan Society for the Promotion of Science (JSPS) KAKENHI Grant No. $15 \mathrm{H} 05702$ is gratefully acknowledged. S. K. was supported by the Basic Research Laboratory Program through the National Research Foundation of Korea (NRF) funded by the MSIT(NRF-2018R1A4A1020696, and NRF-2019R1C1C1010345). A.-M. P. is also supported by Institut Teknologi Bandung through P3MI-ITB 2020 research grant. Computations were performed at Research Institute for Information Technology, Kyushu University. 


\section{Author contributions}

S.K. and T.O. designed the research, K.Y. fabricated the superlattices, W.S.H. carried out the device fabrication, XRR and electrical measurements, W.S.H, K.K., S.K. and T.O. analysed experimental data. A.M.P, S.R. and K.N. carried out and analysed the DFT calculations. H.W. and A.M.P. wrote the manuscript under supervision of S.K. and T.O. with input from all the authors.

\section{Competing interests}

The authors declare no competing interests.

\section{Additional information}

Extended data is available for this paper at https://doi.org/.

Supplementary information is available for this paper at https://doi.org/.

Correspondence and requests for materials should be addressed to S.K. \& T. O.

Reprints and permissions information is available at www.nature.com/reprints. 